\title{
Simplified expressions of the $T$-matrix integrals for electromagnetic scattering
}

\author{
Walter R. C. Somerville, Baptiste Auguié, and Eric C. Le Ru* \\ The MacDiarmid Institute for Advanced Materials and Nanotechnology, School of Chemical and Physical Sciences, \\ Victoria University of Wellington, P.O. Box 600, Wellington 6140, New Zealand \\ *Corresponding author: eric.leru@vuw.ac.nz
}

Received July 15, 2011; revised August 10, 2011; accepted August 15, 2011; posted August 16, 2011 (Doc. ID 151105); published September 1, 2011

\begin{abstract}
The extended boundary condition method, also called the null-field method, provides a semianalytic solution to the problem of electromagnetic scattering by a particle by constructing a transition matrix ( $T$-matrix) that links the scattered field to the incident field. This approach requires the computation of specific integrals over the particle surface, which are typically evaluated numerically. We introduce here a new set of simplified expressions for these integrals in the commonly studied case of axisymmetric particles. Simplifications are obtained using the differentiation properties of the radial functions (spherical Bessel) and angular functions (associated Legendre functions) and integrations by parts. The resulting simplified expressions not only lead to faster computations, but also reduce the risks of loss of precision and provide a simpler framework for further analytical work. @ 2011 Optical Society of America

OCIS codes: $290.0290,290.5825,290.5850$.
\end{abstract}

The extended boundary condition method (EBCM), also called the null-field method, is arguably one of the most elegant and efficient methods to rigorously solve problems of electromagnetic scattering by particles of arbitrary size [1-6]. It can be viewed as a generalization of Mie theory [5] (which applies only to spheres), to the case of particles of arbitrary shape. Since its introduction by Waterman more than 40 years ago [7], it has been successfully applied to a variety of electromagnetic problems. A large part of the work has focused on the far-field properties of homogeneous dielectric particles of intermediate size (typically between $\lambda$ and $20 \lambda$, where $\lambda$ is the wavelength) and with symmetry of revolution $[5,8]$. However, the applicability of the EBCM has been shown to be much wider, including for example nonaxisymmetric scatterers $[9,10]$, scattering by metallic nanoparticles [11-14], and calculations of surface fields $[11,13,14]$. A variant of the method is also widely used in the context of acoustic scattering [15]. Extensions have moreover been proposed, for example, to model nonspherical multilayered (e.g., core-shell) particles [16], collections of particles [17], or high aspect ratio particles using multiple discrete sources [18]. Recently, the problem of calculating the near-field within this framework has also been addressed [19]. The EBCM method can also be used as the basis for further analytical investigations, for example, to formally derive Debye series for arbitrary-shaped particles [20]. Despite these successes, the implementation of the EBCM remains numerically challenging [21], especially for large aspect ratio particles. For example, it has been shown that quadruple precision arithmetic is necessary in some cases [22]. In practice, most of the computing time is spent calculating a large number of surface integrals involving spherical Bessel functions and spherical harmonics, which are used to compute the elements of the $T$-matrix. All scattering characteristics (such as the electromagnetic field everywhere in space) can then be derived. In this Letter, we show that the differentiation properties of the spherical Bessel functions and associated Legendre functions, together with integration by parts, result in drastic simplifications in these integrals. We thereby obtain a new set of simplified integral expressions that will speed up any numerical implementation of the method. These expressions are also more suited to further analytical work using the EBCM formalism.

We only give here a brief outline of the principle of the EBCM, using the notations and conventions of Mishchenko et al. (see [5] for further details) with minor modifications for clarity of presentation. Within the $T$ matrix formulation, the electromagnetic field solutions are expanded in a basis of vector spherical harmonics in a similar fashion as for Mie theory. The expansion coefficients for the scattered field can be represented as vectors $\left(p_{\nu}, q_{\nu}\right)$, where the index $\nu=(m, n)$ and $|m| \leq$ $n$ combines the total $(n)$ and projected $(m)$ angular momentum indices. These coefficients are linearly related to those of the incident field $\left(a_{\nu}, b_{\nu}\right)$ by the $T$-matrix as $\left(\begin{array}{l}\mathbf{p} \\ \mathbf{q}\end{array}\right)=\mathbf{T}\left(\begin{array}{l}\mathbf{a} \\ \mathbf{b}\end{array}\right)$, where $\mathbf{T}$ is an infinite square matrix. Most electromagnetic modeling methods should in principle be able to derive the $T$-matrix (with appropriate truncation), but the EBCM is the only one that provides a semianalytical solution for arbitrary shaped particles. Within this approach, the $T$-matrix is obtained as a matrix product $\mathbf{T}=-\mathrm{Rg}_{\mathbf{Q}} \mathbf{Q}^{-1}$, where the matrix $\mathbf{Q}$ expresses the null-field condition relating the incident and internal fields, while $\operatorname{Rg} \mathbf{Q}$ describes the formation of the scattered field from the internal field. The matrix elements of $\operatorname{Rg} \mathbf{Q}$ and $\mathbf{Q}$ are formally similar and involve integrals of products of vector spherical harmonics over the particle surface. They differ only in the type of spherical Bessel function used: regular for $\operatorname{RgQ}$ and Hankel of the first type for $\mathbf{Q}$. We will focus in the following on $\mathbf{Q}$, but the results are equally valid for RgQ by interchanging the spherical Hankel functions with their regular counterparts. $\mathbf{Q}$ can be expressed as a block matrix

$$
\mathbf{Q}=\left(\begin{array}{ll}
\mathbf{Q}^{11} & \mathbf{Q}^{12} \\
\mathbf{Q}^{21} & \mathbf{Q}^{22}
\end{array}\right)=-i k_{1}^{2}\left(\begin{array}{ll}
s \mathbf{J}^{21}+\mathbf{J}^{12} & s \mathbf{J}^{11}+\mathbf{J}^{22} \\
s \mathbf{J}^{22}+\mathbf{J}^{11} & s \mathbf{J}^{12}+\mathbf{J}^{21}
\end{array}\right)
$$


where $s=n_{2} / n_{1}$ is the relative refractive index of the particle and $k_{1}=2 \pi n_{1} / \lambda$ is the wave vector in the embedding medium. The matrix elements of the $J$-matrices are the surface integrals of interest in this work. In the case of axisymmetric particles, the double integration simplifies to a single integral and the surface integrals are in addition decoupled for different $m$ values, i.e., $J_{m n m^{\prime} k}^{i j}=$ 0 if $m \neq m^{\prime}$. For a given $m$, they can then be expressed in concise form as

$$
\begin{gathered}
\frac{i s k_{1}^{2} J_{n k}^{11}}{A_{n} A_{k}}=\int_{0}^{\pi} \mathrm{d} \theta \sin \theta \xi_{n} \psi_{k}\left[\pi_{n} \tau_{k}+\tau_{n} \pi_{k}\right] \\
\frac{i s k_{1}^{2} J_{n k}^{22}}{A_{n} A_{k}}=\int_{0}^{\pi} \mathrm{d} \theta \sin \theta\left\{\xi_{n}^{\prime} \psi_{k}^{\prime}\left[\pi_{n} \tau_{k}+\tau_{n} \pi_{k}\right]\right. \\
\left.+\left[n(n+1) \xi_{n} \psi_{k}^{\prime}+\frac{k(k+1)}{s} \xi_{n}^{\prime} \psi_{k}\right] \frac{x_{\theta}}{x^{2}} \pi_{n} d_{k}\right\} \\
\frac{s k_{1}^{2} J_{n k}^{12}}{A_{n} A_{k}}=\int_{0}^{\pi} \mathrm{d} \theta \sin \theta \psi_{k}\left\{\xi_{n}^{\prime}\left[\pi_{n} \pi_{k}+\tau_{n} \tau_{k}\right]\right. \\
\left.+\frac{x_{\theta}}{x^{2}} n(n+1) \xi_{n} d_{n} \tau_{k}\right\} \\
\frac{-s k_{1}^{2} J_{n k}^{21}}{A_{n} A_{k}}=\int_{0}^{\pi} \mathrm{d} \theta \sin \theta \xi_{n}\left\{\psi_{k}^{\prime}\left[\pi_{n} \pi_{k}+\tau_{n} \tau_{k}\right]\right. \\
\left.+\frac{x_{\theta}}{s x^{2}} k(k+1) \psi_{k} \tau_{n} d_{k}\right\}
\end{gathered}
$$

where $x(\theta)=k_{1} r(\theta)$ is the angular dependent size parameter $(r(\theta)$ defines the axisymmetric particle geometry in spherical coordinates), $x_{\theta} \equiv \mathrm{d} x / \mathrm{d} \theta$, and $A_{n}=\sqrt{(2 n+1) /(2 n(n+1))}$. We have, moreover, used the shorthand notations

$$
\begin{gathered}
\xi_{n} \equiv \xi_{n}(x(\theta)) \quad \text { and } \quad \xi_{n}^{\prime} \equiv \xi_{n}^{\prime}(x(\theta)), \\
\psi_{n} \equiv \psi_{n}(s x(\theta)) \quad \text { and } \quad \psi_{n}^{\prime} \equiv \psi_{n}^{\prime}(s x(\theta)), \\
\pi_{n} \equiv \pi_{m n}(\theta), \quad \tau_{n} \equiv \tau_{m n}(\theta), \quad \text { and } \quad d_{n} \equiv d_{0 m}^{n}(\theta),
\end{gathered}
$$

where the angular functions $\pi_{m n}, \tau_{m n}$, and $d_{0 m}^{n}$ are defined as in [5]. These integral expressions are essentially identical to those given by Mishchenko et al. [5] , except for the use of the Riccati-Bessel functions instead of the spherical Bessel for simplicity, namely, $\psi_{n}(x)=x j_{n}(x)$ and $\xi_{n}(x)=x h_{n}^{(1)}(x)$.

In order to simplify these expressions, we have used the following properties of the angular functions:

$$
\begin{aligned}
& {\left[\pi_{n} \tau_{k}+\tau_{n} \pi_{k}\right] \sin \theta=m\left(d_{n} d_{k}\right)^{\prime}, } \\
& m d_{n}(\theta=0)= m d_{n}(\theta=\pi)=0 \\
& {\left[\pi_{n} \pi_{k}+\tau_{n} \tau_{k}\right] \sin \theta=}\left(\tau_{k} d_{n} \sin \theta\right)^{\prime} \\
&+k(k+1) d_{n} d_{k} \sin \theta
\end{aligned}
$$

$$
\begin{aligned}
{\left[\pi_{n} \pi_{k}+\tau_{n} \tau_{k}\right] \sin \theta=} & \left(\tau_{n} d_{k} \sin \theta\right)^{\prime} \\
& +n(n+1) d_{n} d_{k} \sin \theta .
\end{aligned}
$$

Moreover, for the radial functions we have

$$
\xi_{n}^{\prime \prime}(x)=\left[\frac{n(n+1)}{x^{2}}-1\right] \xi_{n}(x)
$$

and similarly by replacing $\xi$ by $\psi$ and $x$ by $s x$. From this, we also deduce

$$
\begin{aligned}
& {\left[n(n+1) s^{2}-k(k+1)\right] x_{\theta} \xi_{n} \psi_{k}} \\
& =\frac{\mathrm{d}}{d \theta}\left[k(k+1) \xi_{n}^{\prime} \psi_{k}-n(n+1) s \xi_{n} \psi_{k}^{\prime}\right] \\
& \quad+[n(n+1)-k(k+1)] s x_{\theta} \xi_{n}^{\prime} \psi_{k}^{\prime} .
\end{aligned}
$$

All the preceding relations are easily derived from standard properties of these functions [23].

Focusing first on $\mathbf{J}^{11}$ [Eq. (2)], one can show using Eq. (8), integrating by parts, and using Eq. (9) to remove the nonintegral term, that its matrix elements can be expressed as

$$
\frac{i s k_{1}^{2} J_{n k}^{11}}{A_{n} A_{k}}=\left(-K_{n k}^{2}-s K_{n k}^{1}\right)
$$

where

$$
\begin{aligned}
K_{n k}^{1} & =\int_{0}^{\pi} \mathrm{d} \theta m d_{n} d_{k} x_{\theta} \xi_{n} \psi_{k}^{\prime}, \\
K_{n k}^{2} & =\int_{0}^{\pi} \mathrm{d} \theta m d_{n} d_{k} x_{\theta} \xi_{n}^{\prime} \psi_{k} .
\end{aligned}
$$

Similarly for $\mathbf{J}^{22}$, using Eq. (8) on the first part of Eq. (3), integrating by parts, and using Eqs. (9) and (12), drastic simplifications occur and we obtain

$$
\frac{i s k_{1}^{2} J_{n k}^{22}}{A_{n} A_{k}}=\left(s K_{n k}^{2}+K_{n k}^{1}\right) .
$$

Combining Eqs. (14) and (17), we therefore obtain remarkably simpler expressions for $\mathbf{Q}^{12}$ in term of $\mathbf{K}^{1}$ only and $\mathbf{Q}^{21}$ in terms of $\mathbf{K}^{2}$ only, explicitly:

$$
\begin{aligned}
& Q_{n k}^{12}=A_{n} A_{k} \frac{s^{2}-1}{s} K_{n k}^{1}, \\
& Q_{n k}^{21}=A_{n} A_{k} \frac{1-s^{2}}{s} K_{n k}^{2} .
\end{aligned}
$$

Such dramatic simplifications were not found in the case of $\mathbf{J}^{12}$ and $\mathbf{J}^{21}$, but we can nevertheless obtain alternative expressions by substituting Eq. (10) in Eqs. (4) and (11) in Eq. (5), and integrating by parts to obtain after simplification

$$
\frac{s k_{1}^{2} J_{n k}^{21}}{A_{n} A_{k}}=\left(-s L_{n k}^{1}+L_{n k}^{3}\right),
$$




$$
\frac{s k_{1}^{2} J_{n k}^{12}}{A_{n} A_{k}}=\left(L_{n k}^{2}-L_{n k}^{4}\right)
$$

where

$$
\begin{aligned}
L_{n k}^{1} & =\int_{0}^{\pi} \mathrm{d} \theta \sin \theta x_{\theta} \tau_{n} d_{k} \xi_{n} \psi_{k}, \\
L_{n k}^{2} & =\int_{0}^{\pi} \mathrm{d} \theta \sin \theta x_{\theta} d_{n} \tau_{k} \xi_{n} \psi_{k}, \\
L_{n k}^{3} & =\int_{0}^{\pi} \mathrm{d} \theta \sin \theta d_{k} \psi_{k}^{\prime}\left[x_{\theta} \tau_{n} \xi_{n}^{\prime}-n(n+1) d_{n} \xi_{n}\right], \\
L_{n k}^{4} & =\int_{0}^{\pi} \mathrm{d} \theta \sin \theta d_{n} \xi_{n}^{\prime}\left[s x_{\theta} \tau_{k} \psi_{k}^{\prime}-k(k+1) d_{k} \psi_{k}\right] .
\end{aligned}
$$

Using Eqs. (10), (11), and (13) and integration by parts, we moreover obtain for off-diagonal terms $(n \neq k)$

$$
s L_{n k}^{3}-L_{n k}^{4}=\frac{s^{2} n(n+1)-k(k+1)}{n(n+1)-k(k+1)}\left[L_{n k}^{1}-L_{n k}^{2}\right]
$$

Combining these results, we obtain a simpler expression for the off-diagonal elements of $\mathbf{Q}^{11}$ and $\mathbf{Q}^{22}$ :

$$
\begin{gathered}
Q_{n k}^{11}=\frac{i A_{n} A_{k}\left(s^{2}-1\right) / s}{n(n+1)-k(k+1)}\left[n(n+1) L_{n k}^{2}-k(k+1) L_{n k}^{1}\right], \\
Q_{n k}^{22}=i A_{n} A_{k}\left(s^{2}-1\right) / s\left[L_{n k}^{3}+\frac{s n(n+1)\left(L_{n k}^{2}-L_{n k}^{1}\right)}{n(n+1)-k(k+1)}\right] .
\end{gathered}
$$

Using Eqs. (18), (19), (27), and (28), we can therefore obtain the $Q$-matrix (except its diagonal) by computing the integrals of only five matrices: $\mathbf{K}^{1}, \mathbf{K}^{2}, \mathbf{L}^{1}, \mathbf{L}^{2}$, and $\mathbf{L}^{3}$. We note that the expected vanishing of these matrix elements for $s=1$ is much more obvious in the new expressions than in the original ones. Moreover, these five new integral expressions can all be cast in the separable form $\int f_{n}(\theta) g_{k}(\theta) \mathrm{d} \theta$, which can dramatically speed up numerical computations (compared to an expression like $\left.\int f_{n, k}(\theta) \mathrm{d} \theta\right)$. A similar approach with the original expression of the $J$ matrices would require the computation of 12 such integrals in separable form. In addition to the gain in computation speed and simplicity, the use of simplified expressions dramatically reduces the risks of catastrophic cancellations when subtracting integrals that are very close in value but not equal. For example, the computation of $\mathbf{Q}^{12}$ from $\mathbf{J}^{11}$ and $\mathbf{J}^{22}$ requires the addition of six integrals in separable form. The derivation of Eq. (18) explicitly shows that many of these terms cancel each other, potentially creating catastrophic loss of precision. For a cylinder with parameters $n_{2}=0.09+4 i$ (silver) in water $\left(n_{1}=1.33\right)$, radius $20 \mathrm{~nm}$ and height $80 \mathrm{~nm}$, and an incident wavelength of $633 \mathrm{~nm}$, results from both the double-precision code of Mishchenko et al. [5] and our expressions were compared to arbitrary precision results. Agreement to within an order of magnitude for $\mathbf{Q}^{12}$, $m=1$, was found up to $n_{\max }=35$ for the original expressions, while for our expressions this was extended to $n_{\max }=43$. Finally, for the diagonal elements of the $Q$-matrix, one may also use Eqs. (20) and (21), but there is no real simplification compared to the original expressions [Eqs. (4) and (5)].

In conclusion, we have introduced a new set of simplified integral expressions to compute the surface integrals within the EBCM framework applied to bodies of revolution. These expressions are likely to simplify the numerical implementation of the method, reduce the computing time, and minimize the risks of catastrophic cancellations resulting in loss of precision, which then tends to cause numerical problems for the matrix inversion. These simplifications are particularly spectacular in case of the $\mathbf{Q}^{12}$ and $\mathbf{Q}^{21}$; the computation is reduced to that of a single integral rather than the sum of up to six of them. These new expressions will also simplify further analytical work that uses the EBCM formalism.

The authors are indebted to the Royal Society of New Zealand for support through a Marsden Grant (W. R. C. Somerville and E. C. Le Ru) and a Rutherford Discovery Fellowship (E. C. Le Ru).

\section{References}

1. P. C. Waterman, Phys. Rev. D 3, 825 (1971).

2. P. Barber and C. Yeh, Appl. Opt. 14, 2864 (1975).

3. P. W. Barber and S. C. Hill, Light Scattering by Particles: Computational Methods (World Scientific, 1990).

4. L. Tsang, J. A. Kong, and K.-H. Ding, Scattering of Electromagnetic Waves (Wiley, 2000).

5. M. I. Mishchenko, L. D. Travis, and A. A. Lacis, Scattering, Absorption and Emission of Light by Small Particles, 3rd ed. (Cambridge University, 2002).

6. A. Doicu, T. Wriedt, and Y. A. Eremin, Light Scattering by Systems of Particles: Null-field Method with Discrete Sources: Theory and Programs, Springer Series in Optical Sciences (Springer, 2006), Vol. 124.

7. P. C. Waterman, Proc. IEEE 53, 805 (1965).

8. M. I. Mishchenko, G. Videen, V. A. Babenko, N. G. Khlebtsov, and T. Wriedt, J. Quant. Spectrosc. Radiat. Transfer 88, 357 (2004).

9. J. B. Schneider and I. C. Peden, IEEE Trans. Antennas Propag. 36, 1317 (1988).

10. F. Kahnert, J. Stamnes, and K. Stamnes, Appl. Opt. 40, 3110 (2001).

11. R. Boyack and E. C. Le Ru, Phys. Chem. Chem. Phys. 11, 7398 (2009).

12. B. N. Khlebtsov and N. G. Khlebtsov, J. Phys. Chem. C 111, 11516 (2007).

13. P. W. Barber, R. K. Chang, and H. Massoudi, Phys. Rev. B 27, 7251 (1983).

14. P. W. Barber, R. K. Chang, and H. Massoudi, Phys. Rev. Lett. 50, 997 (1983).

15. P. C. Waterman, J. Acoust. Soc. Am. 45, 1417 (1969).

16. B. Peterson and S. Ström, Phys. Rev. D 10, 2670 (1974).

17. B. Peterson and S. Ström, Phys. Rev. D 8, 3661 (1973).

18. A. Doicu and T. Wriedt, Optics Commun. 139, 85 (1997).

19. A. Doicu and T. Wriedt, J. Quant. Spectrosc. Radiat. Transfer 111, 466 (2010).

20. F. Xu, J. A. Lock, and G. Gouesbet, Phys. Rev. A 81, 043824 (2010).

21. D. Petrov, Y. Shkuratov, and G. Videen, Opt. Lett. 32, 1168 (2007).

22. M. Mishchenko and L. Travis, Optics. Commun. 109, 16 (1994).

23. M. Abramowitz and I. A. Stegun, eds., Handbook of Mathematical Functions (Dover, 1972). 